\title{
What's new in prostate cancer research? Highlights of GU-ASCO 2014
}

Cite as: Can Urol Assoc J 2014;8(3-4Suppl2):S8-12. http://dx.doi.org/10.5489/cuai.2013

Published online April 14, 2014.

\section{Abstract}

At the 2014 Genitourinary Cancers Symposium (GU-ASCO 2014), international researchers presented an extensive array of research in the field of prostate cancer treatment. The following pages provide a summary of some of the most compelling results presented during the 3-day symposium.

\section{Approved novel agents targeting the androgen pathway}

At GU-ASCO 2014, there was a significant body of research presented involving several novel therapies targeting the androgen pathway for the treatment of castration-resistant prostate cancer (CRPC). This included several important studies involving two approved agents, the androgen-synthesis inhibitor abiraterone and the androgen-receptor antagonist enzalutamide.

\section{Enzalutamide}

A pivotal study presented at GU-ASCO 2014 was the Multinational Phase 3, Randomized, Double-Blind, PlaceboControlled Efficacy and Safety Study of Oral MDV3100 in Chemotherapy-Naive Patients With Progressive Metastatic Prostate Cancer Who Have Failed Androgen Deprivation Therapy (PREVAIL) study investigating the utility of enzalutamide in chemotherapy-naïve patients with CRPC. ${ }^{1}$ The study population included a total of 1717 men, enrolled in 22 countries, including Canada. The subjects were randomized 1:1 to enzalutamide capsules $160 \mathrm{mg} /$ day $(\mathrm{n}=872)$ or placebo $(\mathrm{n}=845)$. Concomitant steroids were allowed, but were not required. The co-primary efficacy endpoints were overall survival (OS) and radiographic progression-free survival (rPFS). The trial was intended to continue until there were 765 deaths, with a pre-planned interim analysis planned at 516 deaths (twothirds of the final number of events).

The baseline characteristics were well-balanced in the 2 groups. Median age was 71 years in the placebo group and 72 in the enzalutamide group. Approximately two-thirds of patients in each group were either asymptomatic or mildly symptomatic at initial diagnosis and approximately two-thirds of each group had an Eastern Cooperative Oncology Group (ECOG) performance score of 0 . Approximately $4 \%$ of each group were taking corticosteroids at baseline.

At the time of the interim analysis, the Data Monitoring Committee reported statistically significant benefits in both OS and rPFS in favour of the enzalutamide arm. Because of these findings, the trial was halted and unblinded at that time; the planned interim analysis therefore became the trial's final analysis. Patients from the placebo arm were subsequently offered treatment with enzalutamide.

The median duration of treatment among patients in the enzalutamide arm was 16.6 months, almost 4 times higher than that of the placebo group (4.6 months). At the time of the trial's discontinuation, $42.1 \%$ of enzalutamide patients remained on treatment, as did $7.2 \%$ of those in the placebo arm.

Figures $1 \mathrm{a}$ and $1 \mathrm{~b}$ show the curves for the 2 co-primary endpoints. For OS, enzalutamide significantly reduced the risk by $29 \%$ (hazard ratio [HR] 0.706, 95\% confidence interval [Cl] 0.60 to $0.84, p<0.0001)$. Enzalutamide also prolonged mean rPFS, with an HR of 0.186 (95\% Cl 0.15 to $0.23, p<0.0001)$. The estimated mean rPFS was 3.9 months with placebo $(95 \% \mathrm{Cl} 3.7$ to 5.4 ) and was not yet reached (NYR) with enzalutamide (95\% Cl 13.8 to NYR). For both co-primary endpoints, the results were consistent across pre-specified subgroups (e.g., ECOG 0 or 1, age above or below 75 years, presence or absence of visceral disease and geographic regions). Overall, the subgroup analyses were consistent with the benefit observed for the primary analysis of OS and PFS. Subsequent therapies were used less frequently in the enzalutamide arm than in the placebo arm: $56.7 \%$ of the placebo group was subsequently treated with docetaxel and $45.6 \%$ with abiraterone. In the enzalutamide group, $32.8 \%$ went on to receive docetaxel and $20.5 \%$ received abiraterone.

Objective response rates (complete response [CR] plus partial response [PR], defined as per RECIST 1.1 criteria) were $58.8 \%$ $(19.7 \%+39.1 \%)$ for enzalutamide and $4.9 \%(1 \%$ and $3.9 \%)$ for placebo $(p<0.0001)$. Enzalutamide delayed the median time to chemotherapy by 17 months relative to placebo $(p<0.0001)$.

Serious adverse events (SAEs) were experienced by $32.0 \%$ of patients in the enzalutamide group and $26.8 \%$ of the placebo group. Discontinuations due to adverse events (AEs) were $5.6 \%$ of the enzalutamide group and $6.0 \%$ of the placebo group. AEs leading to death occurred in $4.2 \%$ of the enzalutamide group and $3.8 \%$ of the placebo group. The most common AEs in both groups were fatigue, back pain, constipation and arthralgia. 


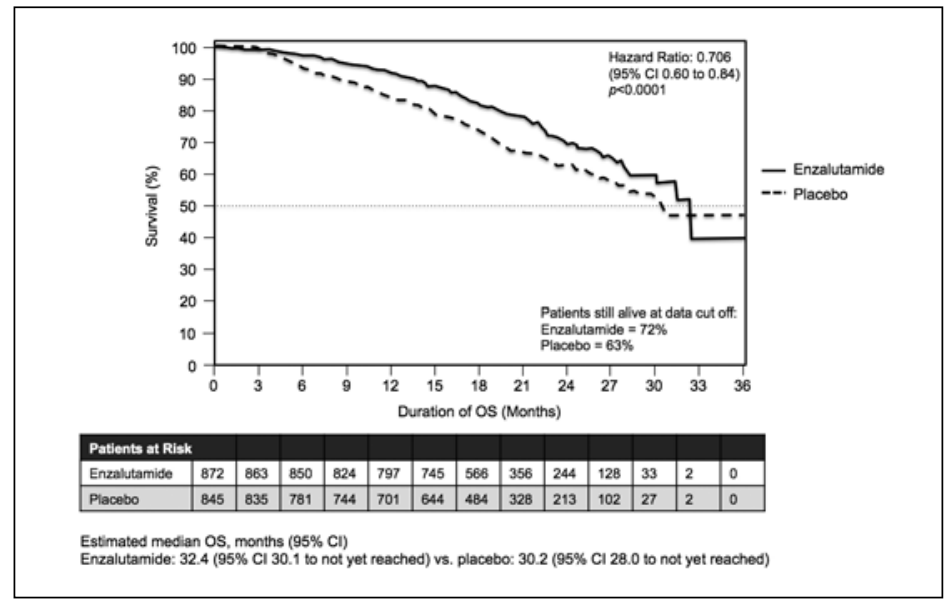

Fig. 1a. Overall survival. PREVAIL study: Co-primary endpoint results: Enzalutamide versus placebo in chemotherapy-naïve patients with castration-resistant prostate cancer $(n=1717)$.

One seizure was reported in the placebo group, and 1 seizure event was subsequently reported in the enzalutamide arm after the date of data cut-off.

Overall, enzalutamide was well-tolerated compared to placebo; it demonstrated minimal side effects, with an impressive $29 \%$ improvement in OS, and reduced the risk of rPFS by $81 \%$ in those patients who have not received chemotherapy.

Another study of interest with enzalutamide presented at GU-ASCO 2014 was the data from the extended follow-up at 49 weeks of the open-label, single-arm, Phase 2 study in 67 patients with histologically-confirmed hormone-naive prostate cancer requiring hormonal treatment and presenting with non-castrate testosterone levels ( $>230 \mathrm{ng} / \mathrm{dL}$ ), prostate-specific antigen (PSA) $>2 \mathrm{ng} / \mathrm{mL}$, ECOG performance status of 0 , and life expectancy greater than 12 months. ${ }^{2}$ Enzalutamide was associated with large reductions in PSA levels, which were maintained over the duration of the 49-week analysis period in this study. At week 49, the PSA response rate for all patients entering the study was $80.6 \%(54 / 67)$ achieving a PSA decrease of $80 \%$ or greater from baseline. Outcomes for all endpoints at 40 weeks were consistent with those reported at 25 weeks, and no new safety signals were observed.

\section{Abiraterone}

One of the studies focusing on abiraterone presented at GU-ASCO 2014 involved data from the Princess Margaret Cancer Centre in Toronto. Dr. Raya Leibowitz-Amit and colleagues studied the association between abiraterone dose, PSA response and progression-free survival (PFS) by means of a retrospective review of all men receiving abiraterone at their institution from November 2009 to March $2013(n=111){ }^{3}$ Of the 111 patients, 91 had received full dose $(1000 \mathrm{mg}$ in the fasting state), while 20 had received a low dose (250 mg or 500 $\mathrm{mg}$ in the fed state). The investigators reported that there was no

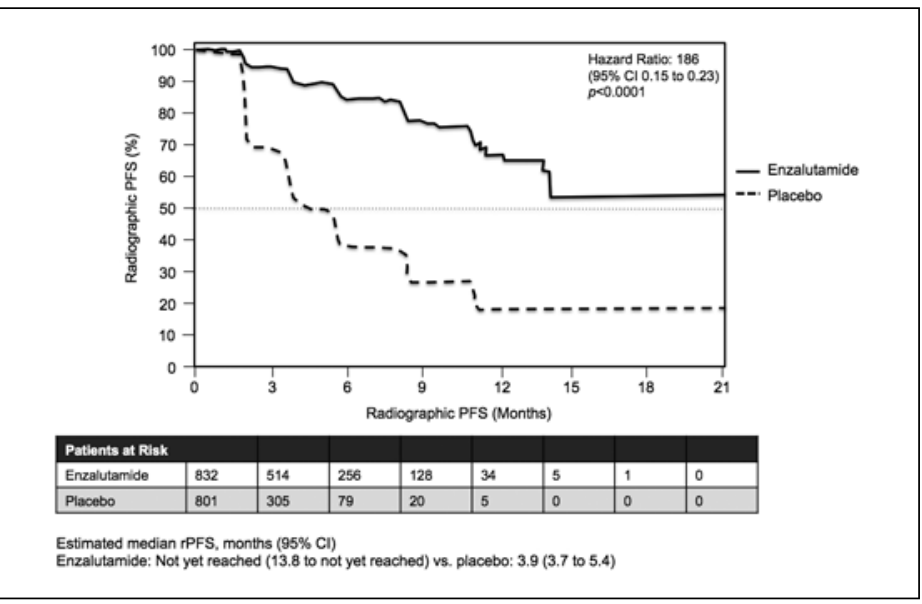

Fig. 1b. Radiographic progression-free survival. PREVAIL study: Co-primary endpoint results: Enzalutamide versus placebo in chemotherapy-naïve patients with castration-resistant prostate cancer $(n=1717)$.

significant difference in PFS or OS between the 2 dose levels in their retrospective analysis. However, the PSA response rates were reported to be non-significantly lower among those treated with low-dose abiraterone, especially in the pre-chemotherapy setting.

\section{Sequencing}

Researchers from the United Kingdom presented the results of their study evaluating the use of enzalutamide among patients who had already received abiraterone therapy. ${ }^{4}$ Given market availability of abiraterone prior to that of enzalutamide, researchers were able to identify 69 patients who had received the agents in this sequence, 57 of whom had received abiraterone as therapy immediately prior to enzalutamide. Among the 69 patients, the investigators concluded that the use of enzalutamide among those who had previously received docetaxel and abiraterone was associated with a prolongation of OS for 11.4 weeks. In $21.7 \%$ of the patients, PFS was ongoing after 36 to 66 weeks of enzalutamide treatment. The median time to progression was 15.1 weeks.

Another retrospective analysis investigating sequencing involved a total of 195 patients treated with enzalutamide at seven academic centres in the United States between January 2009 and August $2013 .{ }^{5}$ The investigators used logistic regression to evaluate the effect of prior abiraterone therapy on the ability to achieve a decline in PSA of $30 \%$ or greater (PSA30) on enzalutamide. Overall, $42 \%$ of enzalutamide-treated patients (76 of 183 evaluable patients) achieved a PSA30. For those who had previously been treated with abiraterone, the PSA30 rate was 39\% (58 of 150 patients). Of the 33 patients who had not previously received abiraterone, the PSA30 rate was $55 \%$ (18 of 33 ). The odds of abirateronenaïve patients achieving a PSA30 were more than twice as high as among abiraterone-experienced patients (odds ratio [OR] 2.3, $95 \% \mathrm{Cl} 1.0$ to $5.5, p=0.06$ ), after adjusting for prior docetaxel 
and concurrent steroid use. This study concludes that a subset of patients with prior abiraterone exposure will have a meaningful PSA decline response to subsequent enzalutamide. Both of these studies highlight the issue of sequencing in CRPC and determining how to best sequence the plethora of novel agents available to optimize outcomes for patients with CRPC. More prospective trials are needed to establish optimal sequencing regimens.

\section{Predicting resistance to novel agents}

At GU-ASCO 2014, Italian researchers presented the results of an analysis that aimed to identify which factor(s), if any, may predict primary resistance to abiraterone or enzalutamide. ${ }^{6}$ The study involved 57 consecutive patients from one institution; 26 received abiraterone and 31 received enzalutamide. Using logistic regression analysis of 24 possible risk factors, those that were predictive of primary resistance in the univariate analysis were: the presence of pain at baseline, high baseline lactate dehydrogenase levels and PSA levels after 1 month of treatment. In multivariate analysis, only PSA levels remained significant as a predictor; patients failing to achieve a reduction of $50 \%$ or greater in baseline PSA levels were more likely to show primary normal human astrocytes (NHA) resistance (48\% vs. $15 \%$, $p=0.007)$. This study is hypothesis-generating and illustrates the need to identify further markers of primary resistance.

\section{Real-world concomitant use of corticosteroids}

A group of Canadian researchers identified 3228 patients treated with abiraterone and 675 patients treated with enzalutamide in an American healthcare database and examined their records to determine the rate of corticosteroid use with these 2 agents. $^{7}$ They reported that the baseline use of corticosteroids prior to initiation of the novel agents was $78 \%$ in the abiraterone cohort and $87 \%$ in the enzalutamide cohort. In the period of active treatment with these drugs, the proportion of patients taking concomitant corticosteroids was $78 \%$ in the abiraterone group and $36 \%$ in the enzalutamide group. The authors concluded that corticosteroid use was common in prostate cancer patients prior to and during treatment with both agents. They recommended further research based on data with longer follow-up and additional observational datasets.

\section{Other novel agents in development}

In addition to the investigations into approved therapies, there were also several intriguing studies documenting the efficacy and safety of other novel agents that are not yet approved in Canada.

\section{Orteronel (TAK700)}

Orteronel (TAK700) is a non-steroidal selective inhibitor of 17,20 -lyase, which is a key enzyme in the production of ster- oidal hormones. At GU-ASCO 2014, researchers presented the results of a Phase 3 study investigating this agent among 1099 men with metastatic CRPC (mCRPC) previously treated with docetaxel (within the previous six months). ${ }^{8}$ Prior abiraterone or ketoconazole was not permitted. Subjects were randomized to receive either continuous 28-day cycles of oral orteronel 400 $\mathrm{mg}$ twice a day plus prednisone $5 \mathrm{mg}$ twice a day $(\mathrm{n}=734)$ or placebo plus prednisone $(n=365)$. The primary endpoint was OS. Other efficacy endpoints included rPFS, 50\% decrease in PSA (PSA50) at 12 weeks and pain response.

After a mean follow-up time of 10.7 months, the study was terminated for failing to meet its primary endpoint. The median OS was 17.0 months $(95 \% \mathrm{Cl} 15.2$ to 19.9$)$ in patients receiving orteronel, which was not significantly different from the median 15.2 months (95\% $\mathrm{Cl} 13.5$ to 16.9$)$ in the placebo arm $(p=0.1898)$ (Fig. 2). However, there were substantial regional differences observed. There was no significant difference between groups in either Europe or North America, but in the rest of the world, the median OS was 15.3 months for orteronel and 10.1 months for placebo (HR $0.71,95 \% \mathrm{Cl} 0.53$ to 0.95 ). Orteronel therapy was associated with an overall improvement in rPFS (median 8.3 months vs. 5.7 months for placebo, $p=$ 0.00038). The most common drug-related AEs were nausea, vomiting, fatigue and diarrhea.

This study illustrates the challenges of reaching statistical significance in OS, despite a significant rPFS as a potential surrogate for OS. Many studies can be confounded by prior and/ or subsequent treatments and crossover; as such, it is suggested that rPFS may be a reasonable surrogate for OS, especially with active novel agents. With orteronel, an overall improvement in rPFS (15.3 months vs. 10.1 months, compared to placebo), translated to a significant OS in regional populations, suggesting that this novel agent has meaningful activity.

\section{Ipilimumab}

Ipilimumab therapy was previously found to have no effect on OS advantage in the Randomized, Double-Blind, Phase 3 Trial to Compare the Efficacy of Ipilimumab versus Placebo in Asymptomatic or Minimally Symptomatic Patients With Metastatic Chemotherapy-Naïve Castration Resistant Prostate Cancer (CA184-043), however there were some indications of anti-tumour activity, including a significant benefit in PFS. At GU-ASCO 2014, researchers presented the results of prespecified subset analyses, which were undertaken to determine whether or not there are features that can identify patients who are more likely to benefit from ipilimumab treatment. ${ }^{9}$ The CA184-043 study involved 799 patients, all of whom received a single dose of radiotherapy and were randomized $1: 1$ to receive either ipilimumab or placebo. As shown in Figure 3, the prespecified subset analyses suggested that ipilimumab may be more active in patients with favourable prognostic factors, including no visceral disease, alkaline phosphatase $<1.5$ upper limit of normal (ULN), and hemoglobin $11 \mathrm{~g} / \mathrm{dL}$. 


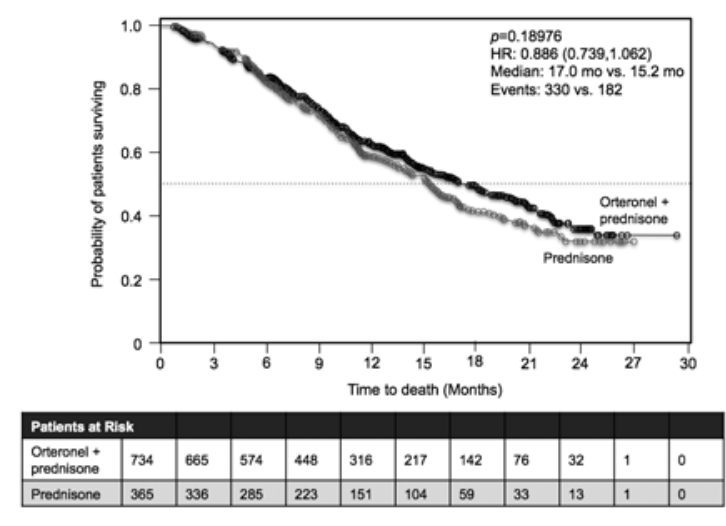

Median follow-up time: 10.7 months (range 0.2-29.5)

Fig. 2. Overall survival with orteronel (TAK700) versus placebo in metastatic castration-resistant prostate cancer.

\section{Galeterone}

Galeterone, a multi-targeted oral steroid analog, works by a combination of androgen receptor modulation (antagonism and degradation) and CYP17 inhibition. At GU-ASCO 2014, researchers presented the first results of a 2-part, Phase 2 Trial of Galeterone in the Treatment of Castration Resistant Prostate Cancer (ARMOR2) with this agent. ${ }^{10}$ For this part of the study, 28 patients were enrolled. Over 12 weeks in this part of the study, the investigators reported 4 Grade 3 AEs, of which both were unrelated to the study agent. In terms of effects on PSA levels, $79 \%$ had reductions of at least $30 \%$ (PSA30) and $67 \%$ of patients achieved a 50\% reduction (PSA50).

\section{Radium-223}

Radium-223 is an alpha-emitting agent that is currently approved in the United States for the treatment of patients with CRPC and bone metastases. The approval of this agent was based primarily on the results of the Double-blind, Randomised, Multiple Dose, Phase 3, Multicentre Study of Alpharadin in the Treatment of Patients With Symptomatic Hormone Refractory Prostate Cancer With Skeletal Metastases (ALSYMPCA), in which OS was prolonged by 3.6 months relative to placebo. At GU-ASCO 2014, the long-term (1.5-year) safety results of ALSYMPCA were presented. ${ }^{11}$ In the safety population, which included 901 patients, there were no major safety issues identified during this period of follow-up. Based upon this favourable safety profile, combination therapy with other novel agents can be considered. The challenge now is how to incorporate radium-223 in the combination/sequencing continuum management of the CRPC patient.

\section{Long-term quality of life in CRPC}

Canadian researchers investigating long-term quality of life (QOL) in high-risk prostate cancer presented the results of their work at GU-ASCO 2014. ${ }^{12}$ The 630 patients included in the Randomized, Multicentre, Phase 3 Study: Assessment of the Duration of Androgen Blockade Combined With Pelvic Irradiation in High-risk Prostate Cancers were randomized to radiotherapy plus 36 or 18 months of androgen deprivation therapy (ADT). The primary efficacy outcome was OS. The QOL was assessed by two validated tools: the European Organisation for Research and Treatment of Cancer 30-item (EORTC30) questionnaire and the prostate-cancer specific 25-item (PR25) questionnaire. The 55 items from the 2 tools were also grouped into 21 scales of related items. The median follow-up was 79 months.

In terms of OS, there was no significant difference between the 18-month and 36-month ADT arms. When comparing the QOL results between the 2 study groups, the 18-month ADT group had significantly better results on six out of 21 scales and 14 out of 55 items. However, the differences were only clinically relevant for 2 of the items (hot flashes and enjoyable sex); none of the differences observed on the scales were deemed to be clinically significant. The investigators concluded that reducing the duration of ADT from 36 to 18 months is associated with some improvements in QOL and has no negative impact on survival.

\section{References}

NB: In addition to the published citations below, information for this article was also taken from the posters and lectures presented at the GU-ASCO 2014 meeting.

1. Beer TM et al. Enzalutamide in men with chemotherapy-naive metastatic prostate cancer (mCRPC): Results of phase III PREVAIL study. J Clin Oncol 2014;32 (suppl 4; abstr LBA1.

2. Tombal $B$ et al. Enzalutamide monotherapy: Extended follow-up of a phase II study in hormone-naive prostate cancer patients. J Clin Oncol 2014;32 (suppl 4; abstr 62).

3. Leibowitz-Amit $R$ et al. Dose-modified abiraterone acetate (AA) in men with metastatic castration-resistant prostate cancer (mCRPC): The Princess Margaret Cancer Centre (PM) experience. J Clin Oncol 2014;32(suppl 4; abstr 61).

4. Stevenson $R$ et al. The sequential use of abiraterone and enzalutamide in metastatic castrate resistant prostate cancer patients: Experience from seven U.K. centers. J Clin Oncol 2014;32 (suppl 4; abstr 125).

5. Cheng HH et al. The effect of prior abiraterone (Abi) use on the activity of enzalutamide (Enza) in men with mCRPC. J Clin Oncol 2014;32(suppl 4; abstr 18).

6. Caffo 0 et al. Looking to possible predictive factors of primary resistance to abiraterone acetate (AA) and enzalutamide (ENZ) in pretreated patients (pts) with castration-resistant prostate cancer (CRPC). J Clin Oncol 2014;32(suppl 4; abstr 248).

7. Lafeuille M-H et al. Real-world corticosteroid utilization in prostate cancer patients treated with two new oral agents. J Clin Oncol 2014;32(suppl 4; abstr 265).

8. Dreicer R et al. Results from a phase 3, randomized, double-blind, multicenter, placebo-controlled trial of orteronel (TAK-700) plus prednisone in patients with metastatic castration-resistant prostate cancer (mCRPC) that has progressed during or following docetaxel-based therapy (ELM-PC 5 trial). J Clin Oncol 2014;32 (suppl 4; abstr 7).

9. Drake CG et al. Results of subset analyses on overall survival (OS) from study CA184-043: Ipilimumab (Ipi) versus placebo (Pbo) in post-docetaxel metastatic castration-resistant prostate cancer (mCRPC). J Clin Oncol 2014;32(suppl 4; abstr 2). 


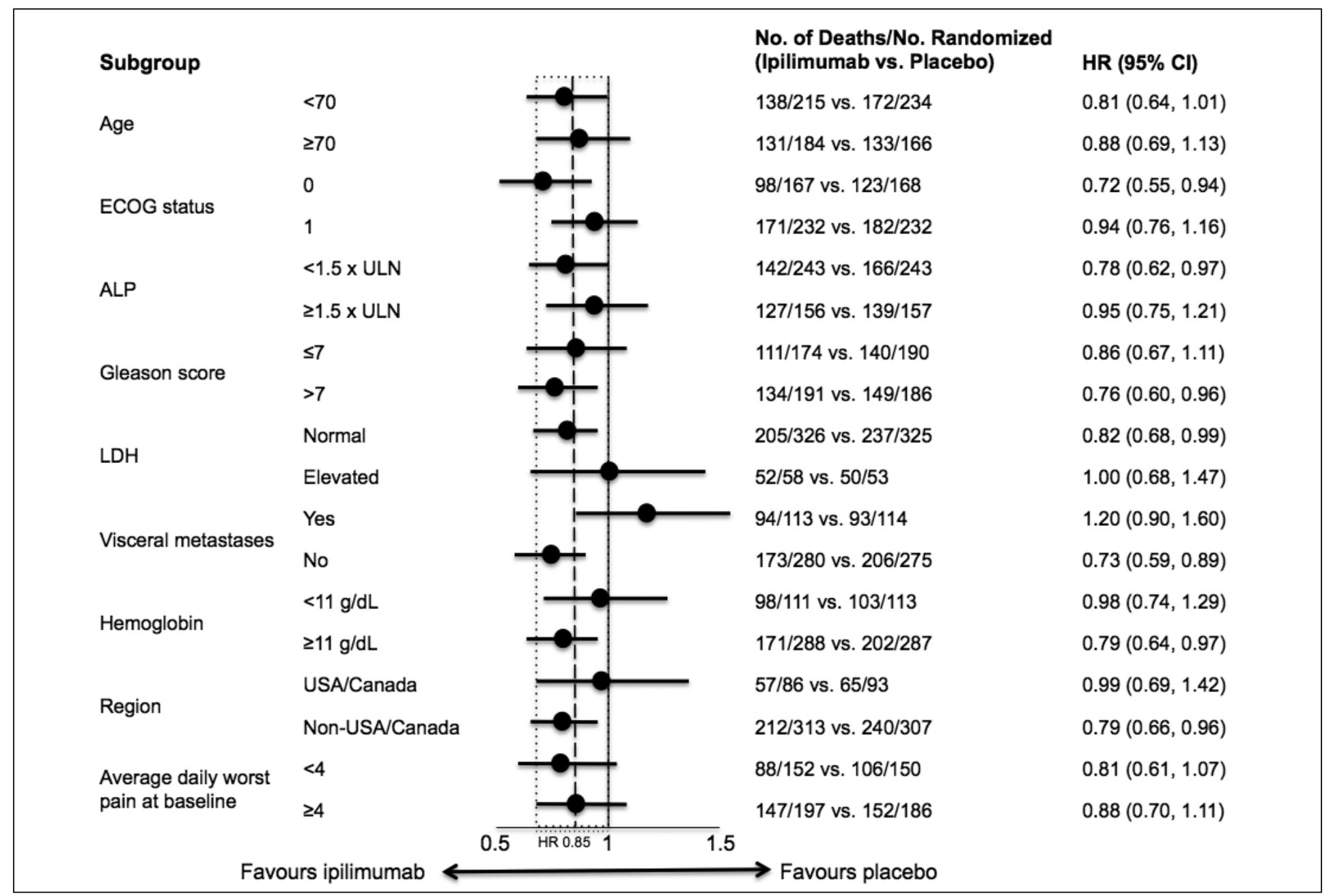

Fig. 3. CA184-043 Trial: Ipilimumab versus placebo in metastatic castration-resistant prostate cancer: Overall survival in pre-specified subgroups.

10. Taplin M-E, Montgomery RB. ARMOR2: Galeterone in progressive CRPC patients who have failed oral therapy. J Clin Oncol 2014;32 (suppl 4; abstr 71).

11. Nilsson $S$ et al. 1.5-year post-treatment follow-up of radium-223 dichloride (Ra-223) in patients with castrationresistant prostate cancer (CRPC) and bone metastases from the phase 3 ALSYMPCA study. J Clin Oncol 2014;32(suppl 4; abstr 9).

12. Nabid A et al. Long-term quality of life in high-risk prostate cancer: Results of a phase III randomized trial. J Clin Oncol 2014;32(suppl 4; abstr 5).
Correspondence: Dr. Anil Kapoor, Professor of Surgery (Urology) and Chair, Genitourinary Oncology Program, Juravinski Cancer Centre, McMaster University, St. Joseph's Hospital, 50 Charlton Ave. E., Hamilton 0N L8N 4A6; akapoor@mmaster.ca 